\title{
A survey of UV-excess AGNs in the South Galactic Pole
}

\author{
A sample for the analysis of the QSO clustering \\ F. La Franca ${ }^{1}$, C. Lissandrini ${ }^{2}$, S. Cristiani ${ }^{2,3}$, L. Miller ${ }^{4}$, M.R.S. Hawkins ${ }^{5}$, and H.T. McGillivray ${ }^{5}$ \\ 1 Dipartimento di Fisica, Università degli studi Roma Tre, Via della Vasca Navale 84, I-00146 Roma, Italy \\ 2 Dipartimento di Astronomia, Università di Padova, Vicolo dell'Oservatorio 5, I-35122, Padova, Italy \\ 3 Space Telescope-European Coordinating Facility, European Southern Observatory, Karl-Schwarzschild Str. 2, D-85748 \\ Garching b. München, Germany \\ 4 Department of Physics, Nuclear and Astrophysics Laboratory, Keble Road, Oxford OX1 3RH, UK \\ ${ }^{5}$ Royal Observatory of Edinburgh, Blackford Hill, Edinburgh EH9 3HJ, UK
}

Received July 8; accepted September 27, 1999

\begin{abstract}
Spectra, position, magnitudes and colors are presented for 485 faint $\left(B_{\mathrm{J}}<20.5\right)$ emission line objects selected with the ultraviolet-excess (UVX) criterion on a area of $24.6 \mathrm{deg}^{2}$ in the South Galactic Pole. The objects were selected from the analysis of pixel-to-pixel stacking of COSMOS scans of UKST $U J$ and $R$ plates. The candidates were observed with the Meudon-ESO Fiber Optics System (MEFOS) at the ESO $3.6 \mathrm{~m}$ telescope. 429 type 1 AGNs have been identified (373 in the redshift range $0.3<z \leq 2.2$ ). This sample has allowed the measure of a difference on the QSO clustering evolution in comparison with that found for galaxies (La Franca et al. 1998). The region is part of the ESO Imaging Survey (EIS) and of the 2dF QSO redshift survey.
\end{abstract}

Key words: catalogs — surveys — quasars: general

\section{Introduction}

In the last decade the study of the evolution of the QSO luminosity function and of the clustering properties of QSOs has been based on statistical analysis of even larger QSO catalogues. For redshifts lower than 2.2 the color techniques provide good selection methods. Now days the

Send offprint requests to: F. La Franca

* Based on material collected with the UK Schmidt Telescope, with the ESO-La Silla Telescopes and on COSMOS scans. Table 3 is only available at the CDS via anonymous ftp to cdsarc.u-strasbg.fr (130.79.128.5) or via http://cdsweb.ustrasbg.fr/Abstract.html and Fig. 1 is only available in the online version of the journal at http://www.edpsciences.org Correspondence to: lafranca@fis.uniroma3.it
QSO luminosity function evolution at $z<2.2$ is well established (e.g. La Franca \& Cristiani 1997; Goldschmidt \& Miller 1998). This kind of studies are mainly based on the most statistically significant QSO samples such us the Durham/AAT QSO sample (Boyle et al. 1990) at faint magnitudes $(B<20.9)$, and the Bright QSO Survey (Schmidt \& Green 1983), the Large Bright QSO Survey (Hewett et al. 1995), the Edinburgh UVX QSO survey (Goldschmidt et al. 1992; Miller et al. in preparation), the Homogeneous Bright QSO Survey (HBQS, Cristiani et al. 1995) at bright magnitudes $(B<18.5)$. In total these samples collect about a thousand of QSOs with $z<2.2$. But these samples are still not ideally suited to study the QSO clustering and its evolution (see Andreani \& Cristiani 1992). The best QSO samples for clustering analysis should have the highest possible surface densities and cover a contiguous areas, such as the planned QSO 2dF redshift survey (Croom et al. 1998).

In order to improve the signal-to-noise ratio in the estimate of the clustering of QSOs, we have built a new sample of 429 QSOs down to $B_{\mathrm{J}}=20.5$ over a contiguous area of $24.6 \mathrm{deg}^{2}$. This sample has allowed the measure of a difference on the QSO clustering evolution in comparison with that found for galaxies (La Franca et al. 1998).

The survey is located in the south Galactic pole (SGP) region, where some of the Durham/AAT sample areas are included (Boyle et al. 1990), and part of the high-redshift QSO survey of Warren et al. (1991b) was carried out. The region has been also studied by Campusano (1991). The central area of $1.7 \mathrm{deg}^{2}$ in our region has been covered by the ESO Imaging Survey in the $B, V$ and $I$ band (EIS, da Costa 1998; Nonino et al. 1999; Prandoni et al. 1999; Zaggia et al. 1999). The acquisition of the corresponding U data is planned for 1999. Further deep multicolor imaging over an area of about half a sq deg is planned with 
the WFI at the ESO $2.2 \mathrm{~m}$ telescope. Moreover about $10 \operatorname{deg}^{2}$ of the southern area of the region is included in the 2dF QSO redshift survey (Croom et al. 1998).

\section{The construction of the catalogue}

Our survey is based on a set of UKST plates in the $U$, $B_{\mathrm{J}}$ and $R$ bandpass (see Blair \& Gilmore 1982 for a definition of the various systems). UKST plates subtend $6.4 \times 6$. 4 . Details of the photographic plate material are listed in Table 1. The plates have been scanned in Mapping and Imaging Mode on the COSMOS microdensitometer (MacGillivray \& Stobie 1984). The mapping mode used a spot size of $16 \mu \mathrm{m}$ (FWHM), corresponding to 1 arcsec. For each band-pass the digitized Mapping Mode data have been added together in order to increase the signal to noise ratio. The reliability of the co-adding technique has been verified by many tests on UKST plates (Hawkins 1991). The resulting coadded digitized data has been analyzed by the COSMOS crowded-field analysis software (Beard et al. 1990). The resulting tables contained the instrumental magnitudes, the area above the threshold, the intensity weighted $x$ and $y$ positions, and other useful parameters. We discriminated between point-like and extended sources using the COSMOS image parameters, that is, on morphological grounds. We defined a plane in which stellar locus was well determined, and drew the separation line near the locus. For typically $B_{\mathrm{J}}<19.5$, we used the $\log$ (isophotal area) versus magnitude plane. At fainter magnitudes, where stellar peak surface brightness $\left(I_{\text {peak }}\right)$ are not saturated, the $\log \left(I_{\text {peak }}-I_{\text {sky }}\right) / I_{\text {sky }}$ versus magnitude plane provided a better separation. The two separation lines produced the same star-galaxy ratio at the overlap magnitude. Relative photometry, using instrumental magnitudes, was produced by COSMOS. Calibration was based on published sequences (Hawkins \& Bessell 1988; Warren et al. 1991a).

The candidates were extracted from a rectangle in the sky centered at $\alpha(1950): 00^{\mathrm{h}} 50^{\mathrm{m}} 34.3^{\mathrm{s}}$ and $\delta(1950):-28^{\circ}$ $10^{\prime} 08^{\prime \prime}$ with limits $\Delta \alpha_{\min }=-2.170^{\circ}, \Delta \alpha_{\max }=2.715^{\circ}$, $\Delta \delta_{\min }=-2.520^{\circ}, \Delta \delta_{\max }=2.520^{\circ}$ (i.e. the limits in right ascension are the two lines distant $\Delta \alpha$ from the meridian at $\alpha(1950)$ : $00^{\mathrm{h}} 50^{\mathrm{m}} 34.3^{\mathrm{s}}$; see Cristiani et al. (1995), their Table 1). All the objects inside a radius of 0.15 degrees centered at $\alpha(1950): 00^{\mathrm{h}} 50^{\mathrm{m}} 17.43^{\mathrm{s}}$, and $\delta(1950)$ : $-26^{\circ} 51^{\prime} 25.2^{\prime \prime}$ have been excluded from the catalogue as this region includes a crowded globular cluster. It results a total area of $24.55 \mathrm{deg}^{2}$.

We have selected as candidates all the UVx "not extremely extended" objects with $B_{\mathrm{J}} \leq 20.5$, satisfying a type of modified Braccesi less-restricted two color criterion (La Franca et al. 1992; Cristiani et al. 1995). The completeness of the selection has been tested against the QSOs already known in the field with redshift in the range $0.3<z \leq 2.2$. We have selected $92 \%$ of the 176 blue QSOs
Table 1. UKST photographic plates

\begin{tabular}{lllcr}
\hline emulsion & filter & number & date & $\begin{array}{r}\text { exp. } \\
\text { time } \\
\text { (min) }\end{array}$ \\
\hline IIaO & UG1 & $U 2639$ & $1976-09-28$ & 180 \\
IIIaJ & UG1 & $U 6326$ & $1980-09-03$ & 180 \\
IIIaJ & UG1 & $U 6380$ & $1980-09-15$ & 180 \\
& & & & \\
IIIaJ & GG395 & $J 9764$ & $1984-11-23$ & 70 \\
IIIaJ & GG395 & $J 9765$ & $1984-11-23$ & 70 \\
IIIaJ & GG395 & $J 9766$ & $1984-11-23$ & 70 \\
IIIaJ & GG395 & $J 9770$ & $1984-11-24$ & 70 \\
IIIaJ & GG395 & $J 9771$ & $1984-11-24$ & 70 \\
& & & & \\
127-04 & RG630 & $R 3498$ & $1977-08-15$ & 90 \\
IIIa-F & RG630 & $R 4676$ & $1978-12-02$ & 90 \\
IIIa-F & RG630 & $R 9594$ & $1984-09-22$ & 90 \\
IIIa-F & RG630 & $R 9672$ & $1984-10-17$ & 90 \\
IIIa-F & RG630 & $R 11331$ & $1986-09-02$ & 90 \\
\hline \hline
\end{tabular}

already known in the field with $B_{\mathrm{J}} \leq 20.5$. The completeness becomes $89 \%$ if the "red" QSOS from Warren et al. (1991a) are included in the comparison.

\section{The spectroscopic survey}

We have used the $3.6 \mathrm{~m}$ ESO telescope, equipped with the Meudon-ESO Fiber Optic System MEFOS (Bellenger et al. 1991), to obtain low-resolution spectra of the QSO candidates in four observational campaigns between 1993 and 1995. MEFOS is a multifibre positioner which enables the conventional use of the ESO Boller \& Chivens Spectrograph to be extended to multiobject spectroscopy. The spectra of up to 29 objects inside the $1^{\circ}$ field of the prime focus of the $3.6 \mathrm{~m}$ telescope can be simultaneously recorded. It consists of 29 remotely controlled arms, each carrying two spectroscopic fibers of 2.5 arcsec of diameter, one for the object, and one for the sky sampling. We used the ESO grating No. 13 (150 grooves $/ \mathrm{mm}$ ) with a resolution of $35 \AA$ in the wavelength range $3600-8400 \AA$. The CCD was a $512 \times 512$ Tektronics with $27 \mu \mathrm{m}$ pixels (ESO No. 32). Wavelength calibration was carried out by comparison with exposures of He and Ar lamps. The campaign dates with the number of pointings and the exposure time are listed in Table 2. The data reduction was performed under MIDAS and followed the optimized spectra extraction from fiber spectrographs of Lissandrini et al. (1994). No absolute flux calibration has been applied. The spectra have been flux calibrated in relative fluxes just for the sake of facilitating the identification. Only the most evident cosmic rays hits have been removed. 
Table 2. The observing log

\begin{tabular}{crl}
\hline Date & $\begin{array}{c}\text { Number } \\
\text { of fields }\end{array}$ & $\begin{array}{l}\text { Integration } \\
\text { time (min) }\end{array}$ \\
\hline 10-Oct.-93/15-Oct.-93 & 15 & $40-70 \times 2$ \\
30-Oct.-94/02-Nov.-94 & 14 & $45-60$ \\
22-Sep.-95/25-Sep.-95 & 8 & $40-50$ \\
22-Nov.-95/25-Nov.-95 & 8 & $60-80$ \\
\hline \hline
\end{tabular}

\section{The catalogue}

Altogether 769 QSOs candidates have been identified (502 from the MEFOS campaign), 485 of which actually turned out to be emission line extragalactic objects. In Fig. 1 the spectra of all the newly identified objects are shown. The $\mathrm{S} / \mathrm{N}$ of the spectra permitted to recognize emissionline objects without leaving much doubt of misidentification. Only 15 objects have a dubious redshift identification. In Table 3 the complete list of the 485 emission line objects and their spectroscopic identification is given. The identification classes are: QSO for broad emission line type 1 AGNs (QSOs and Seyfert 1 galaxies); NL for narrow emission line galaxies (such as Seyfert 2, LINERS, Star-burst or HII emissions). The uncertainty in the redshift estimation is of $0.001 \mathrm{rms}$. At magnitudes brighter than $B<18.7$ the sample is complete and the QSOs are included in the catalogue of the Homogeneous Bright QSO Survey (HBQS, Cristiani et al. 1995). A "d" in the comments identify the objects for which the redshift identification is dubious. A total of 429 type 1 AGNs have been identified, 373 in the redshift range $0.3<z \leq 2.2$. The photometry has an uncertainty of about $0.1 \mathrm{mag}$, while the astrometry has an accuracy of $1^{\prime \prime}$ rms.

MEFOS has been decommissioned by ESO in 1995, and consequently at magnitudes fainter than $B=18.7$ a fraction of the QSO candidates has not been spectroscopically identified. The fainter sample is not useful for statistical applications aimed at measuring the evolution of the luminosity function of AGNs. However, the sample can be used for analysis of the clustering of QSOs by using the technique of scrambling the redshift distribution in the generation of the "random" data set used in the computation of the correlation function (see La Franca et al. 1998).
Acknowledgements. It is a pleasure to thank the enthusiastic support of the COSMOS and UKST staff. The work was partially supported by the Ministry for University and Research (MURST) under grant COFIN98-02-32.

\section{References}

Andreani P., Cristiani S., 1992, ApJ 298, L13

Beard S.M., MacGillivray H.T., Thanisch P.F., 1990, MNRAS 247, 311

Bellenger R., Dreux M., Felenbok P., et al., 1991, The Messenger 65, 54

Blair M., Gilmore G., 1982, PASP 94, 742

Boyle B.J., Fong R., Shanks T., Peterson B.A., 1990, MNRAS 243, 1

Campusano L.E., 1991, A\&A 259, 9

Cristiani S., La Franca F., Andreani P., et al., 1995, A\&AS 112,347

Croom S.M., Shanks T., Boyle B.J., et al., 1998, in Evolution of Large Scale Structure. Garching 1998, astro-ph/9810127

da Costa L.N., 1998, in "Wide Field Surveys in Cosmology", Colombi S., Mellier Y. (eds.), astro-ph/9809209

Goldschmidt P., Miller L., 1998, MNRAS 293, 107

Goldschmidt P., Miller L., La Franca F., Cristiani S., 1992, MNRAS 256, 65

Hawkins M.R.S., 1991, IAU Comm. 9 - Newslett. 1, 23

Hawkins M.R.S., Bessell M.S., 1988, MNRAS 234, 177

Hewett P.C., Foltz C.G., Chaffe F.H., 1995, AJ 109, 1498

Hook I.M., McMahon R.G., Boyle B.J., Irwin M.J., 1994, MNRAS 268, 305

La Franca F., Cristiani S., 1997, AJ 113, 1517

La Franca F., Cristiani S., Barbieri C., 1992, AJ 103, 1062

La Franca F., Andreani P., Cristiani S., 1998, ApJ 497, 529

Lissandrini C., Cristiani S., La Franca F., 1994, PASP 106, 1157

Nonino M., Bertin E., Da Costa L., et al., 1999, A\&AS 137, 43

MacGillivray H.T., Stobie R.S., 1984, Vistas Astron. 27, 433

Prandoni I., Wichmann R., da Costa L., et al., 1999, A\&A 345, 448

Schmidt M., Green R.G., 1983, ApJ 269, 352

Veron-Cetty M.P., Veron P., 1998, ESO Sci. Rep. 18, 1

Warren S.J., Hewett P.C., Irwin M.J., Osmer P.S., 1991a, ApJS 76,1

Warren S.J., Hewett P.C., Osmer P.S., 1991b, ApJS 76, 23

Zaggia S., Hook I., Mendez R., et al., 1999, A\&AS 137, 75 